\title{
Spontaneous Laryngeal Reinnervation Following Chronic Recurrent Laryngeal Nerve Injury
}

\author{
Robbi A. Kupfer, MD; Matthew O. Old, MD; Sang Su Oh, DVM; Eva L. Feldman, MD, PhD; \\ Norman D. Hogikyan, MD
}

Objectives/Hypothesis: To enhance understanding of spontaneous laryngeal muscle reinnervation following severe recurrent laryngeal nerve injury by testing the hypotheses that 1) nerve fibers responsible for thyroarytenoid muscle reinnervation can originate from multiple sources and 2) superior laryngeal nerve is a source of reinnervation.

Study Design: Prospective, controlled, animal model.

Methods: A combination of retrograde neuronal labeling techniques, immunohistochemistry, electromyography, and sequential observations of vocal fold mobility were employed in rat model of chronic recurrent laryngeal nerve injury. The current study details an initial set of experiments in sham surgical and denervated group animals and a subsequent set of experiments in a denervated group.

Results: At 3 months after recurrent laryngeal nerve resection, retrograde brainstem neuronal labeling identified cells in the characteristic superior laryngeal nerve cell body location as well as cells in a novel caudal location. Regrowth of neuron fibers across the site of previous recurrent laryngeal nerve resection was seen in $87 \%$ of examined animals in the denervated group. Electromyographic data support innervation by both the superior and recurrent laryngeal nerves following chronic recurrent laryngeal nerve injury.

Conclusions: Following chronic recurrent laryngeal nerve injury in the rat, laryngeal innervation is demonstrated through the superior laryngeal nerve from cells both within and outside of the normal cluster of cells that supply the superior laryngeal nerve. The recurrent laryngeal nerve regenerates across a surgically created gap, but functional significance of regenerated nerve fibers is unclear.

Key Words: Laryngeal reinnervation, recurrent laryngeal nerve injury, vocal fold paralysis, superior laryngeal nerve, thyroarytenoid muscle, animal model.

Laryngoscope, 123:2216-2227, 2013

\section{INTRODUCTION}

Normal laryngeal physiology requires complex interplay between multiple different components, including sensory input and highly coordinated motor function. Innervation of intrinsic laryngeal muscles is through the superior laryngeal (SLN) and recurrent laryngeal (RLN) branches of the vagus nerve. Motor innervation to all intrinsic muscles except for the cricothyroid muscle (CT)

From the Department of Otolaryngology-Head and Neck Surgery (R.A.K., N.D.H.) and the Department of Neurology (S.S.O., E.L.F.), University of Michigan, Ann Arbor, Michigan; and Department of OtolaryngologyHead and Neck Surgery (m.o.o.), Ohio State University, Columbus, Ohio, U.S.A.

Editor's Note: This Manuscript was accepted for publication January 17, 2013.

This research was supported by the Olympus/American Academy of Otolaryngology-Head and Neck Surgery Foundation Resident Research Grant and the University of Michigan Program for Neurology Research and Discovery. The authors have no other funding, financial relationships, or conflicts of interest to disclose.

This complete study has not been previously presented nor submitted for publication. A portion of this research was presented in a candidate's thesis for fellowship in the Triological Society by Norman D. Hogikyan, MD, and at the Triological Combined Sections meeting in January 2012. The thesis will not be submitted for publication.

Send correspondence to Norman D. Hogikyan, MD, Department of Otolaryngology-Head and Neck Surgery, University of Michigan Medical Center, 1904 Taubman Center, 1500 East Medical Center Drive, Ann Arbor, MI 48109-5312. E-mail: nhogikya@umich.edu

DOI: 10.1002/lary.24049 is thought to occur through the RLN, with the CT being supplied by the external branch of the SLN. ${ }^{1}$ Damage to laryngeal innervation leads to vocal fold paresis or paralysis, for which there currently is no clinical intervention that will reliably restore physiologic movement.

Spontaneous reinnervation of the larynx after RLN injury has been demonstrated in a variety of studies. ${ }^{2-7}$ Investigating the sources of spontaneous laryngeal reinnervation has been the subject of multiple studies in the past 3 decades. ${ }^{3,8-12}$ Review of the existing literature leads logically to three conclusions: 1) the larynx has a propensity for spontaneous reinnervation following RLN injury, 2) reinnervation is a multifactorial process, and 3) spontaneous reinnervation following transecting-type of injuries does not lead to physiologic movement, and reinnervation can be present in chronically immobile vocal folds. The broad long-term goal of our research is to develop techniques that deliver clinical improvement to laryngeal function following nerve injury. A clearer understanding of the processes involved with spontaneous reinnervation is integral to this goal.

Development of the experimental model utilized in the current study and data for control animals were previously published. ${ }^{13}$ In the previous study, methods for rat suspension microlaryngoscopy and endoscopic thyroarytenoid (TA) muscle injection with the retrograde tracer FluoroGold (FG; Fluorochrome, LLC, Denver, 
Colorado) were developed. Anatomic dissections for identification of RLN, SLN, nodose (NOD), superior cervical ganglia (SCG), and dorsal root ganglia (DRG) were refined. Histologic methods for mapping of neurons labeled with FG in brainstem and ganglia were tested. Collection of electromyography (EMG) data was initially pursued during model development, but available needles for insertion were felt at that time to be too traumatic to the TA muscle and therefore potentially a confounder for retrograde labeling experiments with muscle injection. Control retrograde brainstem mapping data were obtained from the TA muscle and for RLN and SLN neurons. Mapping to laryngeal sensory (NOD) and autonomic (SCG) ganglia was also performed.

Retrograde mapping data for the right TA muscle, SLN, RLN, and contamination controls are in the Supplemental Figure. The measurements for brainstem labeling in that figure are distance from the obex in millimeters $(\mathrm{mm})$ on the $\mathrm{x}$ axis and number of cells on the $\mathrm{y}$ axis. Neurons of the RLN mapped consistently to a brainstem distribution with a bimodal peak in the NA and dorsal motor nucleus of the vagus nerve (N10) more caudal than the SLN peak with some overlap, consistent with published data. Injection of the TA muscle with FG yielded a mixed RLN and SLN brainstem distribution demonstrating that the TA is innervated by both nerves, contrary to classical teaching. If both nerves were cut before TA injection, no brainstem labeling occurred; these animals served as negative controls. Ganglia uptake in SCG and NOD was consistent with uptake by mixed motor, sensory, and autonomic fibers in both RLN and SLN, but the SLN had a much greater proportion of sensory and autonomic labeling than the RLN, as expected. No significant uptake was present in DRG for any rats, serving as a negative control for systemic uptake of FG.

The current study was undertaken after we had developed and published a model through previous studies. The aim of this study was to characterize the sources of spontaneous reinnervation in the rat TA muscle following long-term RLN injury. Hypotheses to be tested were 1) that the nerve fibers responsible for TA muscle reinnervation can originate from multiple sources and 2) that the SLN is involved in reinnervation of the TA muscle.

\section{MATERIALS AND METHODS}

\section{Animals}

Procedures were performed under the approval of the University of Michigan Committee on Use and Care of Animals. Initial experiments were carried out using 16 male SpragueDawley rats, age 80 days, divided equally into sham (S) and denervated (D) groups and treated according to the schema in Figure 1. The difference between the eight $\mathrm{S}$ and eight $\mathrm{D}$ group animals was only in the nature of the initial procedure as described in the following animal procedures section; the remaining treatment was the same for animals S1 through S8 and D1 through D8. Upon completion of these experiments and analyses of acquired data, further studies were specifically designed to explore in more detail and with a larger experimental group some of the most compelling initial results. For sake of clarity, this next set of experiments will be referred to in the

\section{INITIAL EXPERIMENTS}

\author{
Sham $(S)(n=8)$ and Denervation $(D)(n=8)$ \\ Groups \\ Initial Surgical Procedure and Endoscopy<smiles>C[13CH2]</smiles> \\ Endoscopy - 1 month \\ $\downarrow$ \\ Endoscopy -2 months \\ $\downarrow$ \\ Endoscopy -3 months \\ $\downarrow$ \\ Rat \#1: Cut R RLN, inject FG R TA muscle \\ \#2: Cut R SLN, inject FG R TA muscle \\ \#3: Cut R RLN, apply FG to nerve end \\ \#4: Cut R SLN, apply FG to nerve end \\ \#5-8: Inject FG R TA muscle \\ $\downarrow$ \\ Perfuse and harvest 6 days later
}

Fig. 1. Schema for surgical procedures and retrograde mapping in the initial experiments. $\mathrm{FG}=$ FluoroGold; $\mathrm{R}=$ right; $\mathrm{RLN}=$ recurrent laryngeal nerve; $S L N=$ superior laryngeal nerve; $\mathrm{TA}=$ thyroarytenoid.

following sections as the subsequent experiments. Twenty-one male Sprague-Dawley rats, age 80 days, were used for these subsequent experiments. Thirteen additional D group animals (D9-D21) were treated according to the schema in Figure 2, and eight other rats were used for development of EMG techniques and to acquire normal and acute denervation EMG data for comparison to experimental animals.

\section{Animal Procedures}

General anesthesia with spontaneous respiration and microlaryngoscopy was performed as previously described. ${ }^{13} \mathrm{In}$ brief, intraperitoneal ketamine $(50 \mathrm{mg} / \mathrm{kg})$ and xylazine $(5 \mathrm{mg} /$ $\mathrm{kg}$ ) with inhaled $1.8 \%$ isoflurane were used to induce anesthesia for endoscopy to document vocal fold mobility. The larynx, trachea, and right RLN were then exposed under an operating microscope through a midline cervical incision. In those rats that were to undergo denervation, $5 \mathrm{~mm}$ of the right RLN was resected. Retrograde labeling procedures were performed according to Figures 1 and 2 using techniques previously published. ${ }^{13}$ Briefly, FG was injected into the right TA muscle of designated animals using endoscopic guidance. Transcervical application of FG to the proximal nerve stump of the RLN or SLN was performed in designated animals. 


\section{SUBSEQUENT EXPERIMENTS}

\section{Denervation $(D)(n=13)$ Group}

\section{Denervation and Initial Endoscopy}

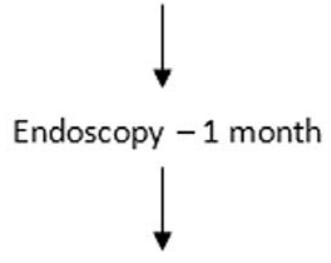

Endoscopy -2 months<smiles>C[13CH2]</smiles>

Endoscopy -3 months<smiles>C[13CH2]</smiles>

Rat \#9-14: Inject FG R TA muscle

\#15-21: Cut R SLN, apply FG to nerve end<smiles>C[13CH2]</smiles>

Perfuse and harvest 6 days later

*Rats \#9, 10, 11, 15, 16, 21: EMG prior to harvest

Fig. 2. Schema for surgical procedures and retrograde mapping for subsequent set of experiments. $E M G$ = electromyography; $\mathrm{FG}=$ FluoroGold; $\mathrm{R}=$ right; $\mathrm{SLN}=$ superior laryngeal nerve $\mathrm{TA}=$ thyroarytenoid

\section{Transoral Laryngeal EMG}

Original previously published model development did not include TA muscle EMG because of concerns about muscle trauma from EMG needles that could affect retrograde labeling. After acquiring initial labeling data in the current studies, however, it was felt that transoral EMG should be pursued in the subsequent experiments to augment information about innervation status of examined muscles. A total of 14 rats underwent transoral laryngeal EMG: six rats from the subsequent experimental group (3 that underwent acute right SLN transection and FG application and 3 that underwent FG injection into the TA muscle), two EMG control rats that had undergone no prior procedures, three rats that underwent acute right RLN resection 7 days before EMG, and three rats that underwent acute right RLN and right SLN resection 7 days before EMG. Rats were anesthetized for microlaryngoscopy with spontaneous respiration as described. Using a 30degree pediatric telescope for visualization, a 26-gauge concentric needle electrode (VIASYS NeuroCare, Madison, WI) was inserted into the TA muscle to measure spontaneous EMG during respiration (Fig. 3). This technique was adapted from Tessema et al. ${ }^{14}$ Spontaneous EMG was recorded for the bilateral TA muscles on all animals. EMG interpretation was performed by one author (E.L.F.), who was blinded to any prior denervation procedures.

\section{Harvest and Tissue Processing}

Rats that underwent FG labeling procedures were euthanized and harvested on postoperative day 6 following tracer

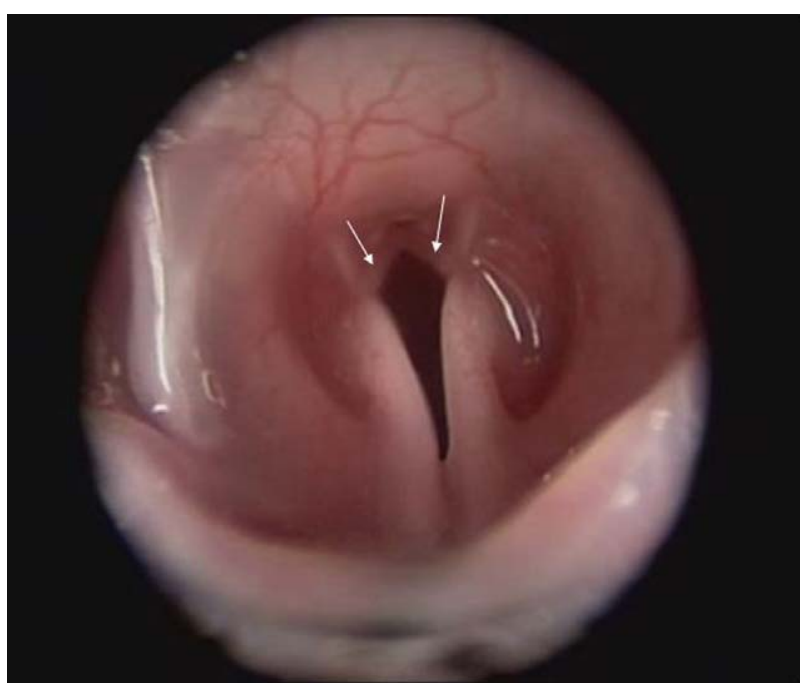

Fig. 3. Endoscopic view of the rat larynx. White arrows indicate electromyography electrode insertion points for the thyroarytenoid muscles.

application, and brainstems, DRG, SCG, and NOD ganglia were immediately dissected and processed as previously described. ${ }^{13}$ ProLong Gold antifade reagent with DAPI (Life Technologies, Grand Island, New York) was used to quantify FG positive cells, which were counted and recorded according to side (left, right) and precise rostral/caudal position in relation to the obex. In the subsequent experimental group, FG positive cells were further characterized by size, morphology, and dorsal/ventral and medial/lateral location to determine in which brainstem nucleus the cells were located. Trachea with associated RLNs were also harvested, sectioned at $20 \mu \mathrm{m}$, and processed with neurofilament stain. This was done to examine the region of prior RLN segmental resection and allow comparison with contralateral normal nerve.

\section{RESULTS}

\section{Animal Procedures}

All 29 experimental animals and eight EMG (control and acute denervation) animals survived to the chosen endpoint, underwent designated procedures, and were successfully harvested. Eighteen endoscopic injections of FG into the right TA muscle were performed with three injections considered technically inadequate (animals S7, D5, and D12).

\section{Vocal Fold Motion Analysis}

All initial RLN resections correlated with immediate ipsilateral vocal fold paralysis (Table I). This includes all $21 \mathrm{D}$-group animals plus the six EMG acute RLN section animals. Four of 21 D-group rats developed partial movement, including rats D21 and D3. During the final FG procedures, rat D21 underwent transection of the SLN, and rat D3 underwent retransection of the proximal RLN. Retransection was performed to eliminate any contribution to reinnervation or movement through regenerated RLN fibers. Interestingly, the partial movement persisted in both of these rats. Three rats (D2, D8, and D20) developed nonpurposeful fibrillation 
TABLE I.

Endoscopic Right Vocal Fold Motion Assessment for Denervated Rats in Primary and Confirmatory Experiments.

\begin{tabular}{|c|c|c|c|c|c|c|}
\hline Group D & 0 - Presurgery & 0 - Postsurgery & 1 Month & 2 Months & 3 Months & 3 Months Postsurgery \\
\hline \multicolumn{7}{|c|}{ Initial experiments } \\
\hline D1 & + & - & M & - & - & - \\
\hline D2 & + & - & $\mathrm{F}$ & - & $\mathrm{F}$ & - \\
\hline D3 & + & - & - & - & M & M \\
\hline D4 & + & - & - & - & - & - \\
\hline D5 & + & - & - & - & - & NA \\
\hline D6 & + & - & - & - & M & NA \\
\hline D7 & + & - & - & - & - & NA \\
\hline D8 & + & - & - & - & $\mathrm{F}$ & NA \\
\hline \multicolumn{7}{|c|}{ Subsequent experiments } \\
\hline D9 & + & - & - & - & - & NA \\
\hline D10 & + & - & - & - & - & NA \\
\hline D11 & + & - & - & - & - & NA \\
\hline D12 & + & - & - & - & - & NA \\
\hline D13 & + & - & - & - & - & NA \\
\hline D14 & + & - & - & - & - & NA \\
\hline D15 & + & - & - & - & - & - \\
\hline D16 & + & - & - & - & - & - \\
\hline D17 & + & - & - & - & - & - \\
\hline D18 & + & - & - & - & - & - \\
\hline D19 & + & - & - & - & - & - \\
\hline D20 & + & - & - & $\mathrm{F}$ & $M$ & $M$ \\
\hline D21 & + & - & $M$ & $M$ & $M$ & $M$ \\
\hline
\end{tabular}

$\mathrm{D}=$ denervated; $\mathrm{F}$ = fibrillation; $\mathrm{M}$ = partial movement; $\mathrm{NA}=$ not applicable; + = full movement; - = paralysis.

of the vocal fold: D2 and D20 subsequently underwent SLN resection; fibrillations ceased in rat D2 after SLN section but remained in rat D20.

\section{Trachea With Associated RLN Histologic Section Analysis}

Immunohistochemical evidence of RLN regrowth across the resection gap by presence of neurofilament was evident in 13 of 15 (87\%) D rats available for tracheal sectioning (Fig. 4). Visual comparison to the intact contralateral side showed regenerated axons were less robust and more disorganized, becoming more scattered as sections progressed distally. In addition, neurofilament was present in the distal nerve stumps, but the distal nerves were partially overtaken by fibrosis. RLN regrowth did not correlate with vocal fold motion.

\section{Retrograde Labeling}

Control data from preliminary studies have been previously published and are presented in the Supplemental Figure. ${ }^{13}$ In those studies and also in current initial experiments, all FG-positive neurons within the brainstem were mapped by their rostral-caudal position, but not to a specific nucleus. In the subsequent experiments, FGpositive neurons were mapped by rostral-caudal position and were also further characterized by location within the nucleus ambiguus (NA) or dorsal motor nucleus of the vagus (N10). N10 cells could be differentiated from NA cells by a more medial and ventral location and smaller size (Fig. 5). There were no FG-positive cells in the DRG of any animal, which served as a negative control.

\section{Initial Experiments: $S$ Group (Fig. 6)}

RLN and SLN mapping. Results for the S-group animals essentially paralleled control data. RLN cell bodies (rat S3) map to a bimodal distribution in the brainstem, with the majority in a peak from the obex to $1 \mathrm{~mm}$ rostral to that point. Ganglia mapping through the RLN labeled to the ipsilateral and contralateral NOD. Cell bodies of the SLN (rat S4) map to a more rostral and narrow region of the brainstem, with a large rostral peak. Ganglia mapping through the SLN labeled much larger numbers of sensory and autonomic neurons.

Right TA muscle injections. Right TA injections demonstrated a pattern consistent with uptake primarily through the RLN with a lesser amount of SLN uptake (rats S4-S8). Ganglia mapping showed large uptake in the ipsilateral NOD, moderate amounts in the contralateral NOD and ipsilateral SCG, and small amounts in the contralateral SCG. When the TA muscle was injected after acute RLN (rat S1) or SLN (rat S2) transection, brainstem mapping showed a small rostral peak (S1) in the SLN distribution and a bimodal peak in the RLN 

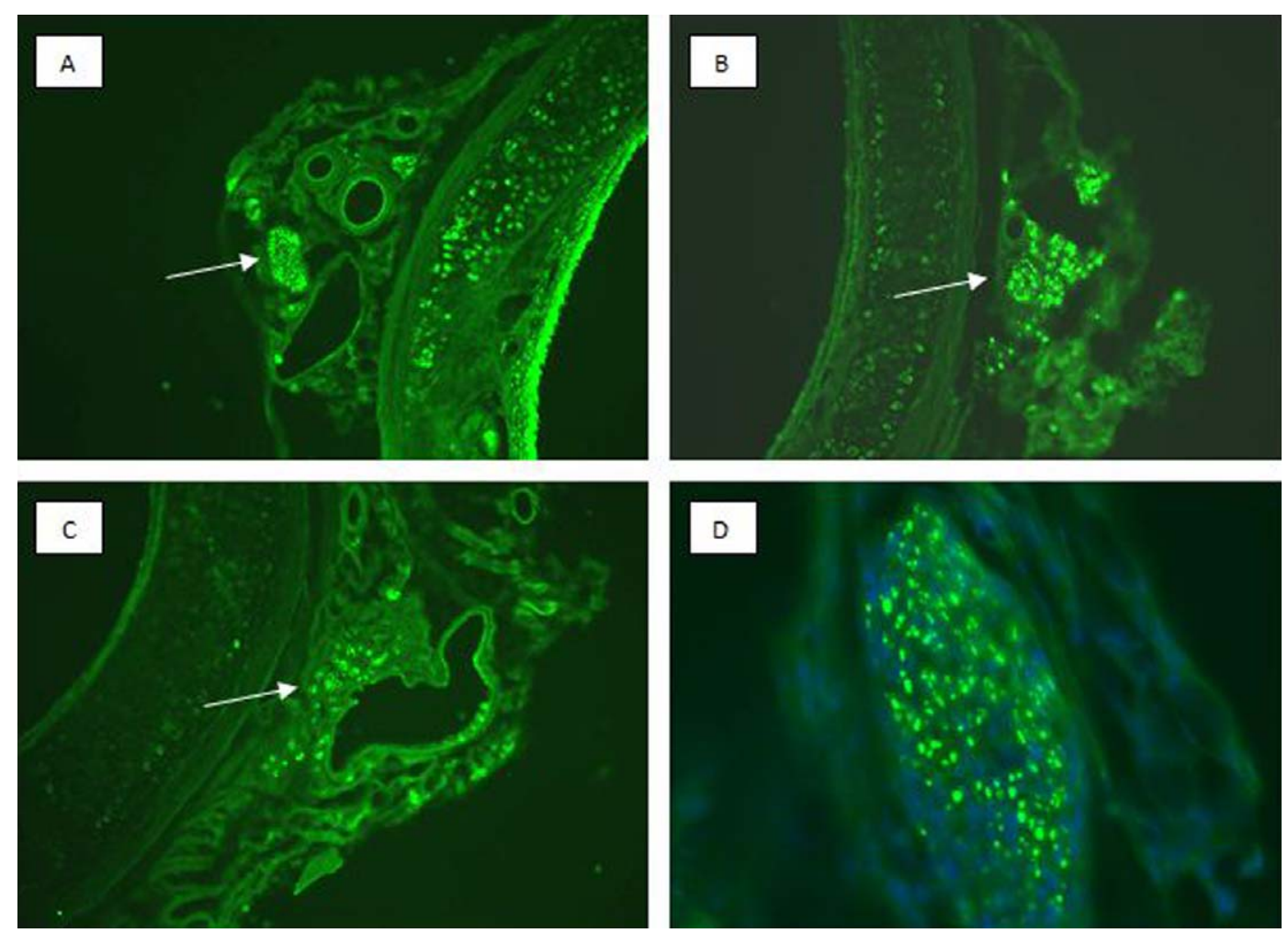

Fig. 4. Histology of recurrent laryngeal nerves (RLNs) with associated trachea. White arrows indicate areas of immunohistochemical staining of neurofilament. (A) Intact left RLN with associated blood vessels. (B) Regrowth of disorganized bundle of neurofilaments across right RLN resection site. (C) Scar with smaller and more disorganized neurofilaments across right RLN resection site. (D) Distal nerve stump costained with 4',6-diamidino-2-phenylindole (blue) demonstrating decreased number of axons within a partially fibrotic nerve.

distribution (S2), consistent with primarily RLN innervation to the TA with secondary SLN innervation.

\section{Initial Experiments: D Group (Fig. 7)}

RLN and SLN mapping. D-group animals yielded markedly different results from the S-group and control animals. Rat D3 underwent retransection of the RLN proximal to the prior nerve resection followed by retrograde labeling through this stump. Retransection was performed to eliminate any contribution through the RLN to reinnervation. This resulted in neurons mapping in reduced numbers to a region overlapping the more rostral area of prior
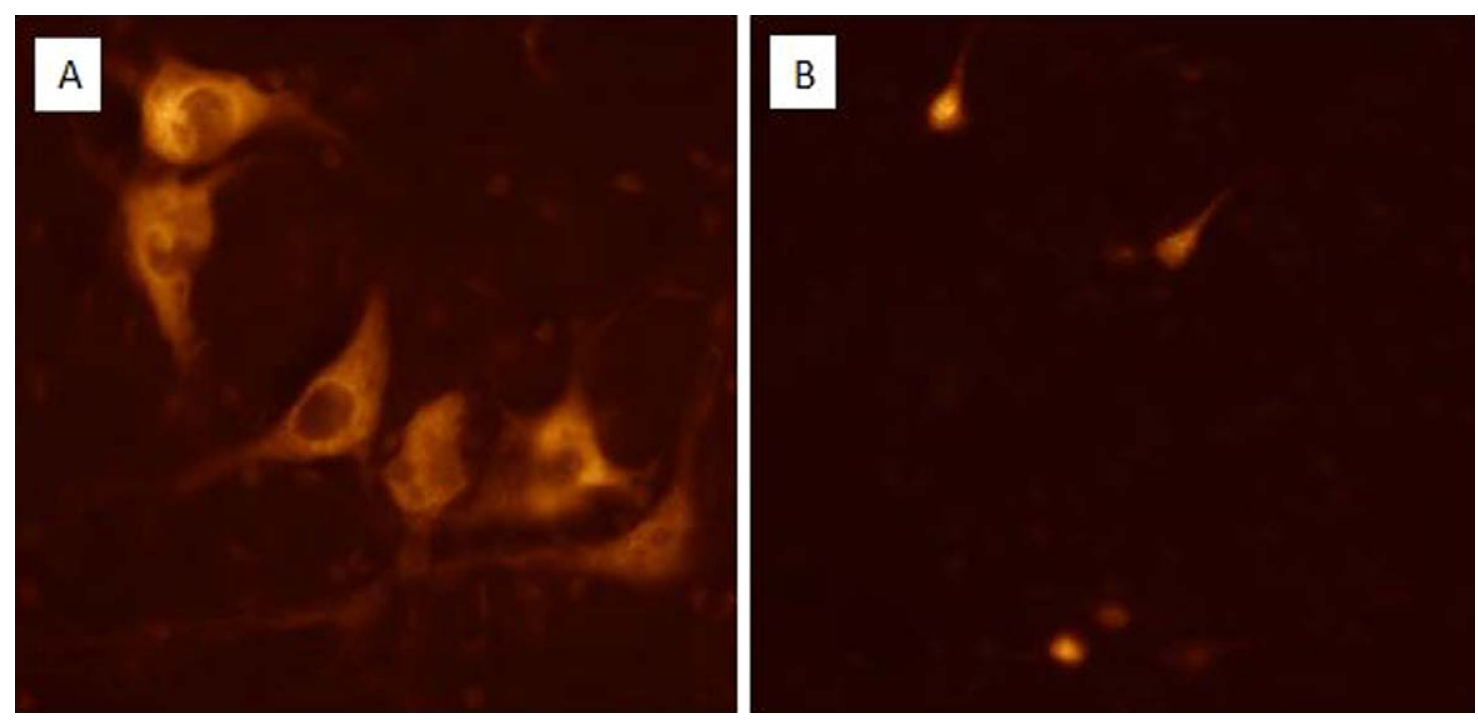

Fig. 5. FluoroGold-positive cells in nucleus ambiguus (A) and dorsal motor nucleus of the vagus (B) at the same magnification. Note the much smaller size of cells within the dorsal motor nucleus of the vagus compared to the nucleus ambiguus. 


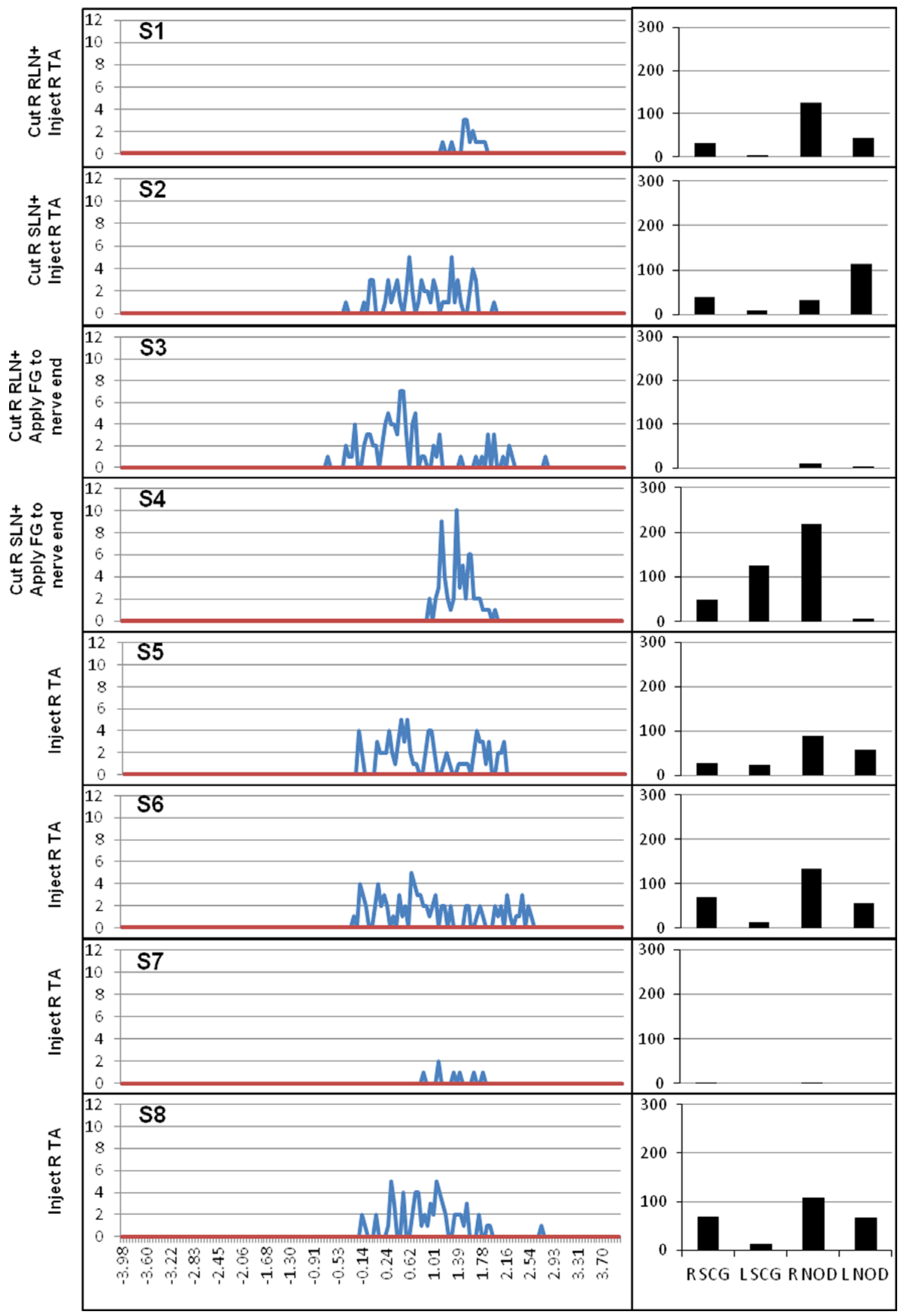

Fig. 6. Retrograde mapping of FluoroGold (FG)-positive cells in the brainstem (first column) and ganglia (second column) for group S. Measurements for brainstem are millimeters from the obex ( $x$ axis) and number of labeled cells $(y$ axis). Blue lines are right-sided cells and red are left. Ganglia are superior cervical (SCG) and nodose (NOD) with number of labeled cells. $L=$ left; $R=$ right; $R L N=$ recurrent laryngeal nerve; SLN = superior laryngeal nerve; TA =thyroarytenoid. [Color figure can be viewed in the online issue, which is available at wileyonlinelibrary.com.] 


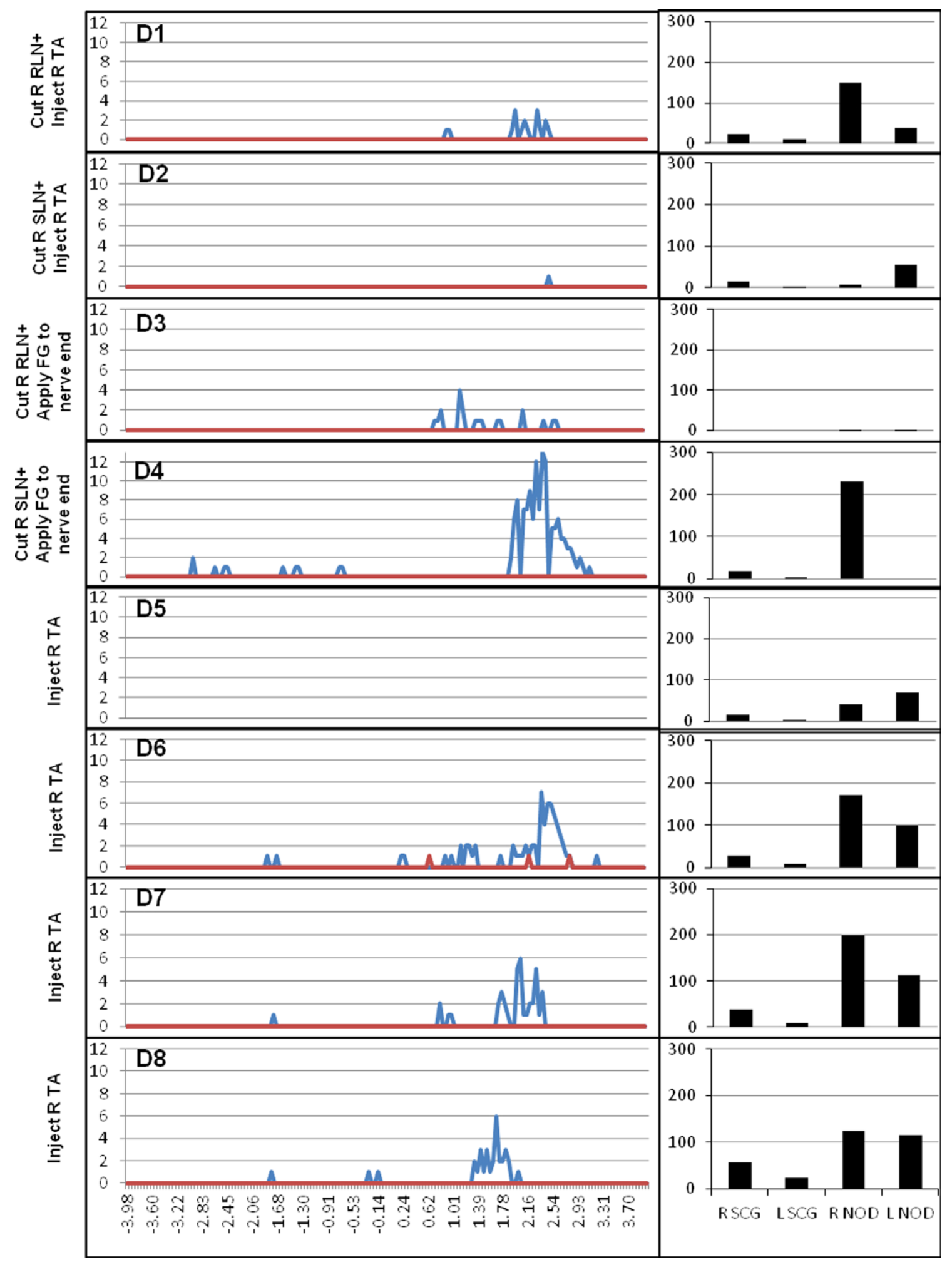

Fig. 7. Retrograde mapping of FluoroGold (FG)-positive cells in the brainstem (first column) and ganglia (second column) for group D from the initial experiments. Measurements for brainstem are millimeters from the obex (x axis) and number of labeled cells ( $y$ axis). Blue lines are right-sided cells and red are left. Ganglia are superior cervical (SCG) and nodose (NOD) with number of labeled cells. $L=$ left; $R=$ right; $\mathrm{RLN}=$ recurrent laryngeal nerve; $\mathrm{SLN}=$ superior laryngeal nerve; $\mathrm{TA}=$ thyroarytenoid. [Color figure can be viewed in the online issue, which is available at wileyonlinelibrary.com.]

RLN mapping, and to the SLN region at 0.7 to $2.6-\mathrm{mm}$ rostral to the obex. There was also minimal mapping in the ipsilateral and contralateral NOD. Mapping of SLN neu- rons (rat D4) labeled cells in a new caudal region not identified in any other groups, as well as a large peak in the rostral brainstem partially overlapping with previously 
identified SLN neurons. Ganglia mapping showed similar findings to S-group and control animals in the ipsilateral NOD and SCG but much less in the contralateral SCG.

Right TA muscle injections. Right TA injections following RLN resection (rats D5-D8) showed a pattern similar to direct labeling through the SLN (rat D4), including cells within the novel caudal region, but reduced in numbers. Ganglia labeling showed a moderate to large amount in the ipsilateral NOD and a moderate amount in the contralateral NOD and both SCG. When the right TA muscle was injected after acute RLN transection (rat D1), there was continued labeling in the region of prior SLN or rostral RLN mapping, but with reduced numbers. Transection of the SLN before right TA injection (rat D2) essentially halted brainstem uptake with only a single labeled cell.

\section{Subsequent Experiments}

Analyses of initial experiments suggested that normal SLN neurons as well as neurons from a novel caudal location were involved with reinnervation of the TA muscle. In particular, paradigms used for animals D4 (labeling through the SLN) and D5-D8 (labeling following right TA muscle injection) were believed to warrant further specific investigation. These subsequent experiments were therefore undertaken to increase the sample size for labeling following TA muscle injection (D9-D14) or directly through the SLN (D15-D21).

Right TA muscle injections. Brainstem mapping showed a similar rostral-caudal pattern for D5 to D8 from the initial experiments having undergone the same procedure (Fig. 8). The majority of FG-positive cells were located in the ipsilateral NA, but two animals
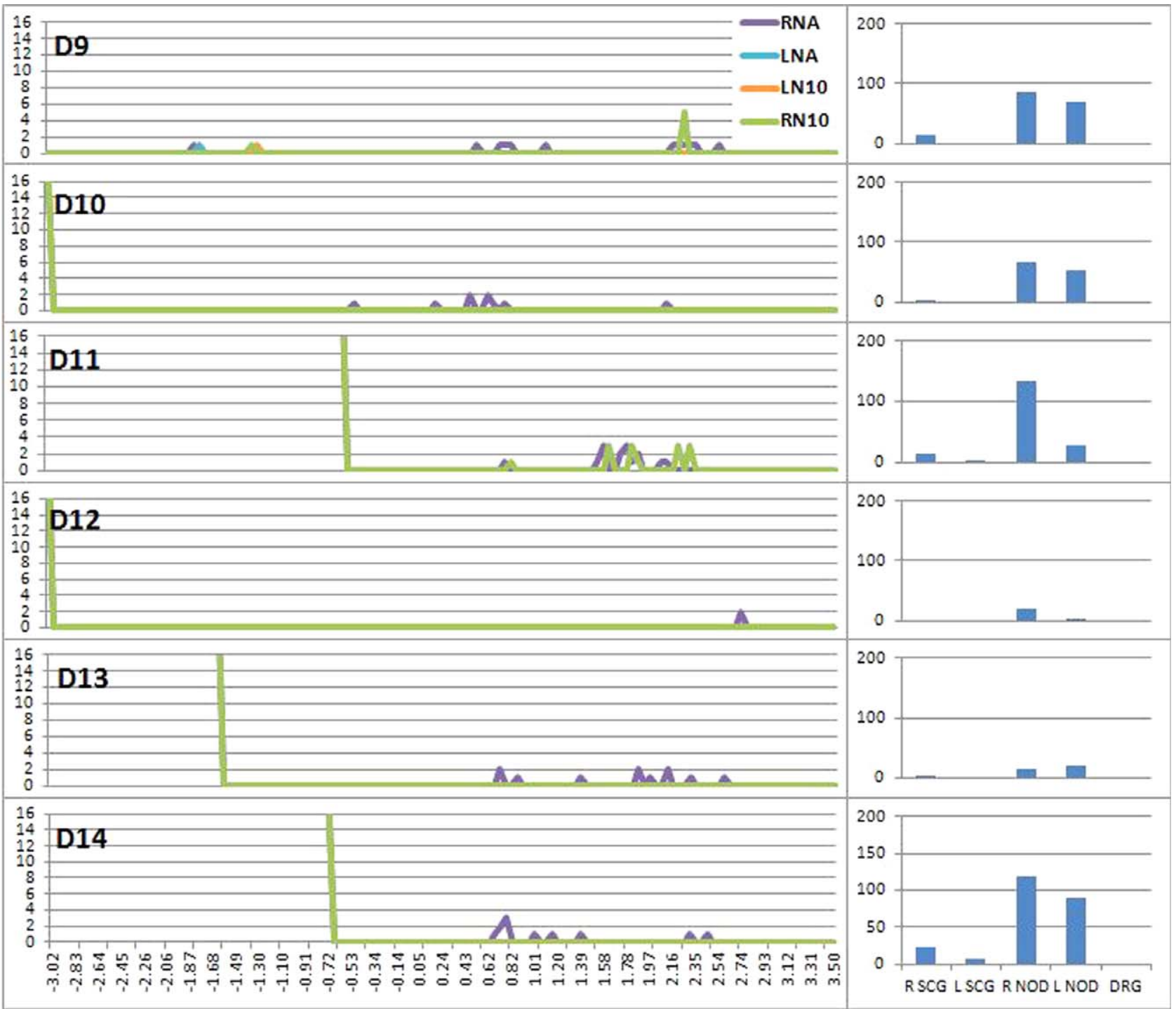

Fig. 8. Retrograde mapping of FluoroGold (FG)-positive cells in the brainstem (first column) and ganglia (second column) in rats from the subsequent experiments that underwent injection of FG into the right thyroarytenoid muscle. Measurements for brainstem are millimeters from the obex ( $x$ axis) and number of labeled cells ( $y$ axis). Vertical green lines indicate the most caudal point at which sections were available. Ganglia are superior cervical (SCG), nodose (NOD), and dorsal root ganglia (DRG) with number of labeled cells. $L=$ left; $L N A=$ left nucleus ambiguus; LN10 = left dorsal motor nucleus of the vagus; RNA = right nucleus ambiguus; RN10 = right dorsal motor nucleus of the vagus. [Color figure can be viewed in the online issue, which is available at wileyonlinelibrary.com.] 
demonstrated a significant proportion of cells that mapped to the ipsilateral $\mathrm{N} 10$ (33\% in rat D9 and $48 \%$ in rat D11). The rostral-caudal location of these N10 cells was similar to the main cluster of cells that mapped to the NA. The novel area of caudal cells that had been identified during the initial experiments in rats D4, D6, D7, and D8 was found only in rat D9, where a single cell from each nucleus was found at that caudal location. However, because of inadvertent tissue processing errors, the caudal extent of evaluation was truncated in several animals (indicated by the vertical green line in Fig. 8), and so it was not possible to assess this region in those animals. Ganglia mapping was similar to rats D5 through D8 in the initial group.

SLN mapping. Overall rostral-caudal position of FG-positive cells was similar to findings in rat D4, with a strong peak in the rostral brainstem (Fig. 9). The previously noted caudal cells were seen in four animals. All of these caudal cells mapped to the right N10. Because of tissue processing, the caudal extent of evaluation was again truncated in several animals (indicated by the vertical green line in Fig. 9). Ganglia mapping was similar to rat $\mathrm{D} 4$, with strong labeling to the ipsilateral NOD and very few cells mapping to the ipsilateral SCG.

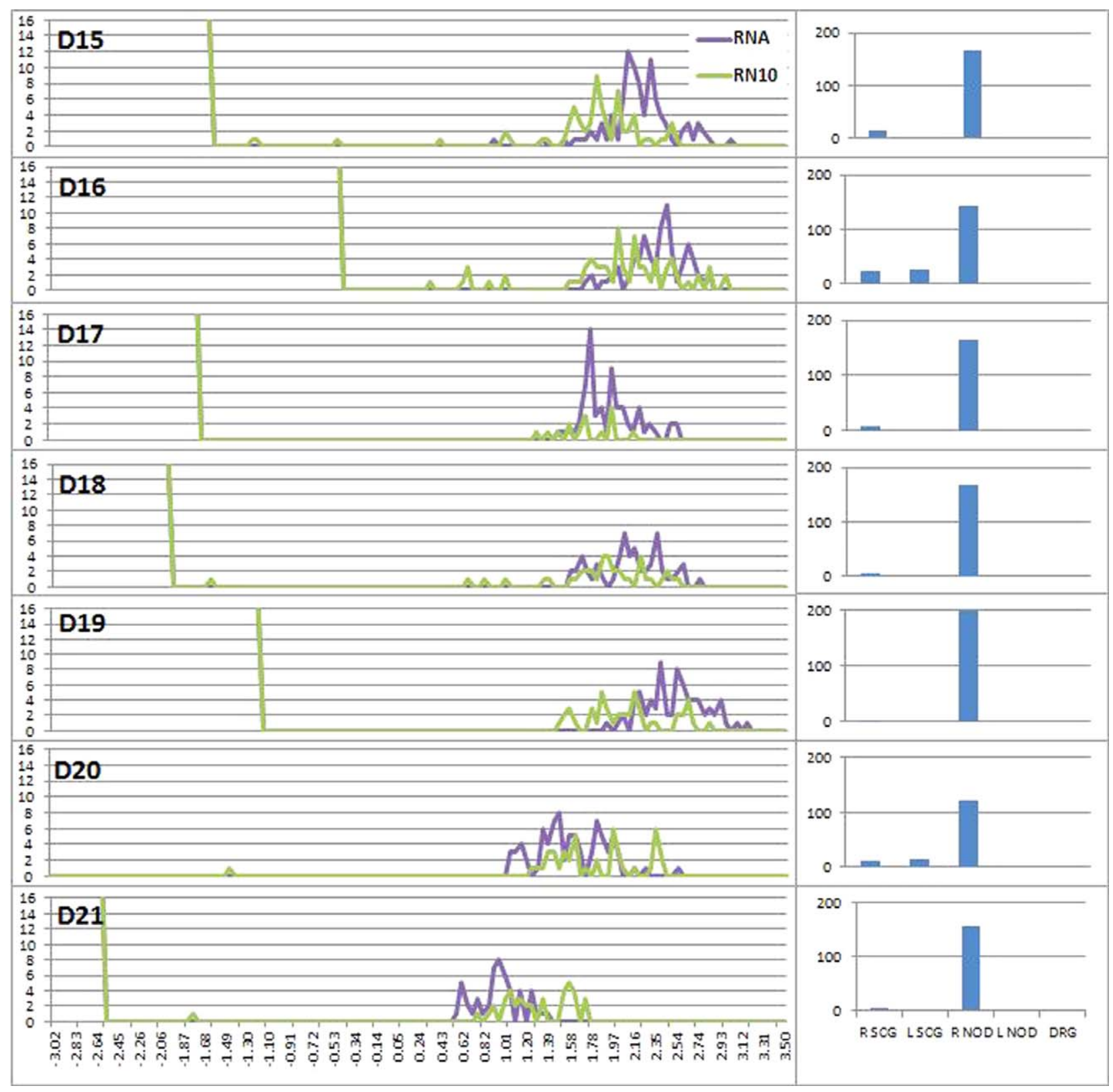

Fig. 9. Retrograde mapping of FluoroGold (FG)-positive cells in the brainstem (first column) and ganglia (second column) in rats from the subsequent experiments that underwent right superior laryngeal nerve transection and application of FG to the cut end. Measurements for brainstem are millimeters from the obex ( $x$ axis) and number of labeled cells ( $y$ axis). Vertical green lines indicate the most caudal point at which sections were available. Ganglia are superior cervical (SCG), nodose (NOD), and dorsal root ganglia (DRG) with number of labeled cells. $L=$ left; $R=$ right; RNA = right nucleus ambiguus; RN10 = right dorsal motor nucleus of the vagus. [Color figure can be viewed in the online issue, which is available at wileyonlinelibrary.com.] 
Laryngeal EMG. EMG recordings from the right TA muscle of chronically denervated rats at 3 months after RLN resection demonstrated observable motor unit action potentials, but with decreased insertional activity compared to the left TA muscle in the same rats. In those rats that underwent chronic right RLN denervation followed by acute right SLN transection for the FG procedures, insertional activity was further decreased compared to those that underwent chronic right RLN denervation alone. Notably, rat D21 showed some recovery of right vocal fold movement on endoscopy and was also found to have nearly normal EMG signal in the right TA muscle at 3 months after RLN resection and 1 week after SLN transection.

Acutely denervated rats demonstrated fibrillation and positive sharp wave potentials in the ipsilateral TA muscle on laryngeal EMG 1 week after denervation. Transection of the RLN and ipsilateral SLN resulted in strong fibrillation and positive sharp wave potentials, but transection of the RLN alone yielded less consistent results, with fibrillation potentials noted in only one of three rats. Vocal fold motion assessment of all acutely denervated rats demonstrated right vocal fold immobility at the time of EMG recording. Control rats that had undergone no denervation procedures and the intact left TA muscle of the D-group rats consistently demonstrated normal motor unit action potentials as well as normal bilateral vocal fold movement. Figure 10 shows examples of EMG recordings from control and D rats.

\section{DISCUSSION}

It is clear from control results presented in a prior paper and current S-group animals that in the normal state, the rat TA muscle receives dual innervation from the RLN and SLN with the RLN predominating. ${ }^{13}$ Dual innervation is contrary to what is taught as textbook laryngeal neuroanatomy, but review of the literature finds this concept previously presented for humans and multiple other species. ${ }^{15-18}$

Retrograde neural labeling directly through laryngeal nerves and following TA muscle injection provides evidence that the SLN is a source of spontaneous reinnervation to the TA muscle in the setting of chronic RLN injury. There is also evidence for central nervous system (CNS) plasticity in that both expected SLN neurons and a small number of neurons outside the normal location of cells projecting axons through the SLN are identified through retrograde labeling. In the subsequent set of experiments from the current study, these caudal cells were characterized both by linear distance from the obex and by their two-dimensional location in brainstem cross-section. Their location appears to be in N10, the parasympathetic nucleus of the vagus nerve. No S-group animals from the current study or control animals from the preliminary studies showed direct SLN labeling in this region.

The exact nature of the novel caudal-labeled cells in the D-group animals cannot be defined with our current data. Recently published evidence shows that multiple forebrain systems converge on lower motor neurons

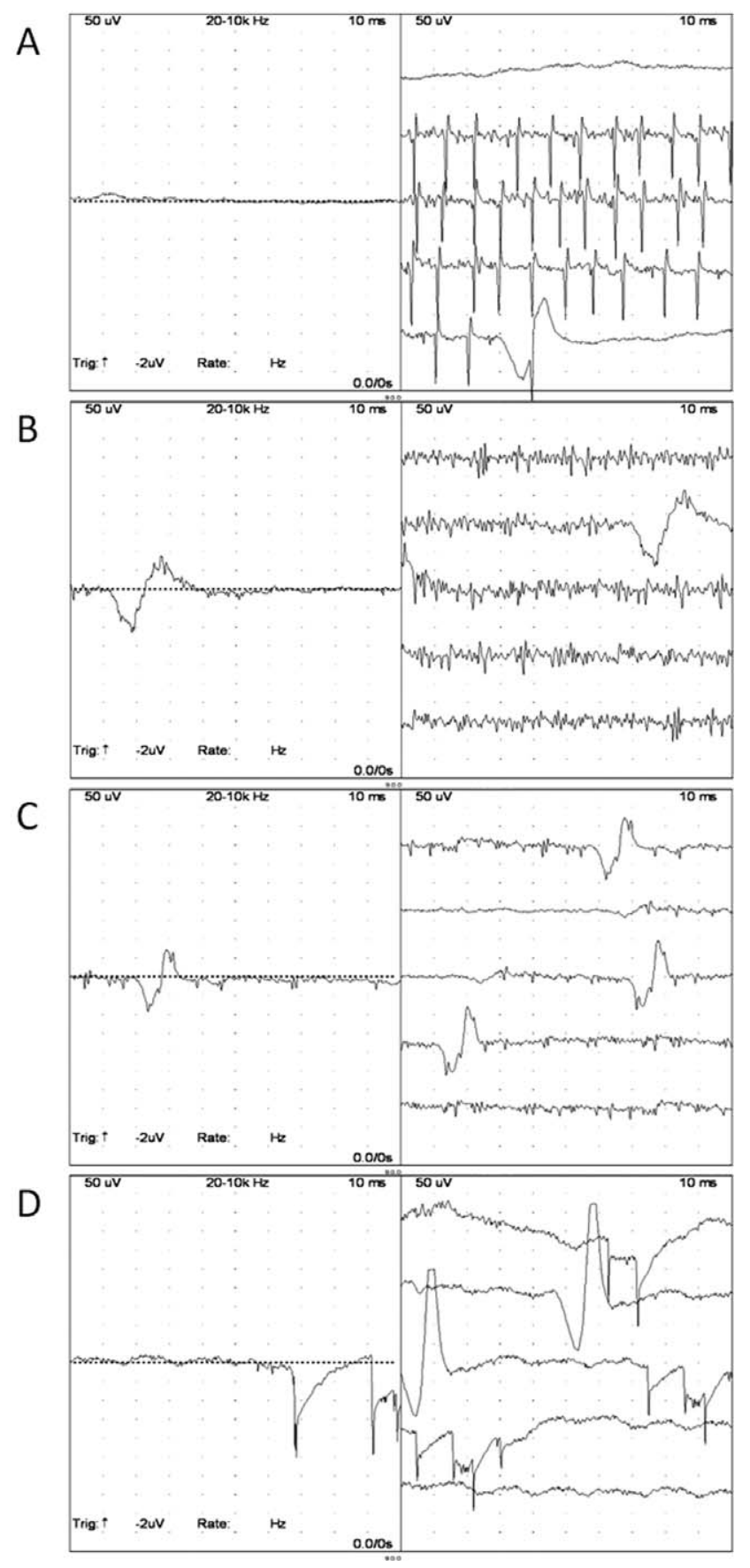

Fig. 10. Electromyography recordings from the thyroarytenoid muscle of rats. (A) Intact innervation, demonstrating observable motor unit action potentials. (B) Chronic recurrent laryngeal nerve $(R L N)$ denervation, demonstrating reinnervation but with decreased insertional activity. (C) Chronic RLN denervation and acute superior laryngeal nerve (SLN) denervation, demonstrating a greater decrease in insertional activity. (D) Acute RLN and SLN denervation, demonstrating fibrillation and positive sharp wave potentials.

innervating the TA muscle. ${ }^{19}$ In addition, expression of nestin, a neural stem/progenitor cell-enriched marker, has been demonstrated in the NA following vagal nerve injury. ${ }^{20}$ This finding is an indication of rejuvenilization of NA neurons in response to peripheral nerve injury 
and a demonstration of CNS plasticity. These studies underscore the points that neural pathways involved with laryngeal innervation are complex and that we are truly just beginning to understand them in the normal and injured states.

Electromyographic data also support the SLN as a source of TA innervation after RLN injury. Rats that underwent chronic RLN resection followed by acute SLN transection as part of FG labeling procedures were found to have decreased insertional activity in the ipsilateral TA muscle compared to those rats that underwent chronic RLN resection alone. Together with the EMG data in acutely denervated rats, these findings suggest that the SLN is a source of innervation of the TA muscle in both the normal and denervated states.

Given prior evidence in the literature for regeneration of the RLN across experimentally created gaps, we examined this phenomenon histologically and found an $87 \%$ rate of regrowth. We were not able to determine definitively whether these axons form functional neuromuscular junctions in the TA or other laryngeal muscles from our current data. However, functional and EMG data from rat D21 showed continued vocal fold movement and observable motor unit action potentials in the setting of chronic RLN injury, even after acute transection of the ipsilateral SLN, suggesting that reinnervation includes sources other than the SLN.

One-third of $\mathrm{D}$ rats in the study developed some degree of right vocal fold mobility during the 3-month course of observation. Perhaps the most interesting findings were in those rats that developed movement and had subsequent nerve-sectioning procedures. Rat D2 underwent section of the SLN as a final procedure before FG injection at 3 months, with subsequent abatement of disorganized vocal fold movements that had been seen previously. However, rats D20 and D21 continued to have partial vocal fold movement, even after undergoing SLN section. Rat D3 had resectioning (to eliminate contributions from regenerated RLN fibers) of the RLN before application of FG to the nerve stump at 3 months, and the partial movement seen in that animal continued. These findings further support the conclusion that there are several sources of reinnervation in the setting of chronic RLN injury.

It would also be logical that with multiple possible reinnervation sources available following RLN injury, the specific pattern of reinnervation could vary significantly from case to case; that is, the relative contribution from each source would not be expected to be uniform. Defining all potential sources and if possible determining favorable ones would be valuable steps toward an ultimate goal of improving clinical outcomes after nerve injury. Strategies to augment favorable sources and/or restrict unfavorable ones could then be employed. McRae et al. have demonstrated in a rat model the ability to prevent laryngeal synkinesis following RLN injury through use of local neurotoxins. ${ }^{21}$

Analyses of sensory (NOD) and autonomic (SCG) ganglia labeling provide additional insights about the makeup of neural components in the RLN and SLN and efferent and afferent innervation of the TA muscle.
When considered together, control data from a prior study and $\mathrm{S}$ data from the current study indicate that the RLN carries a very limited amount of sensory and sympathetic fibers, with ipsilateral sensory (right NOD) being the greatest. In contrast to this, the SLN has a much greater sensory (NOD) component and also more autonomic (SCG) fibers, consistent with the known larger sensory function of the SLN. Notably, there is also bilateral labeling, and to our knowledge the anatomic pathways for this are not well defined.

\section{Limitations of the Current Study}

One limitation of this study is the small sample size for animals undergoing a particular intervention in the primary experiments. The labor-intensive nature of these experiments precluded a large sample size for each intervention and labeling procedure in the initial studies. Therefore, the subsequent experiments were designed to corroborate the most interesting findings from these studies by increasing the number of $\mathrm{D}$ animals undergoing labeling through the SLN and directly from the TA muscle. We also note a potential limitation that the transport of FG through an injured/regenerated nerve may be unreliable. This makes it possible that all neurons reaching the TA through regenerated axons may not be identified through retrograde labeling. During the subsequent set of experiments in the current study, it became apparent that characterizing FG-positive cells by both linear distance from the obex and nuclear location in NA or N10 would be informative. Unfortunately, this differentiation of FG-positive cells into distinct nuclei was not performed in earlier animals; those cells were only described by their rostral-caudal location. Attempts to retrospectively analyze the brainstem slides were unsuccessful due to fading of fluorescent labeling. Another limitation was the inadvertent truncation of the brainstem specimens in several experimental animals, thus limiting evaluation of a caudal region of interest in four rats. This resulted from the inability to accurately identify the rostral-caudal position of the obex grossly during tissue harvest, because the precise location of the obex can only be determined during histologic review.

\section{Implications for Future Research}

Understanding the pathways through which the SLN contributes to laryngeal innervation in the setting of chronic RLN injury warrants further investigation. Previous work has suggested that intramuscular sprouting occurs in this setting, which may be the mechanism of TA muscle reinnervation. ${ }^{12}$ Determining the functionality of regenerated RLN fibers is also an important step in understanding the likely multidimensional process of reinnervation. Further studies are underway in both of these areas.

\section{CONCLUSION}

It is clear that, contrary to what has been taught traditionally in regard to anatomy, the rat TA muscle in 
its normal state receives dual innervation through the RLN and the SLN. In the setting of chronic RLN injury, there are changes in CNS organization of the SLN and reinnervation of widowed fibers in the TA through the SLN by cells that include typical SLN neurons as well as ones outside the normal cluster of medullary cells that supply the SLN. We also conclude that the RLN does have the ability to regenerate axons across a surgically created gap in a high percentage of cases, but the functionality of these regenerated axons is uncertain. The ultimate goal of this research remains the clinical improvement of laryngeal function following nerve injury. Further experimentation to understand the sources and processes involved with spontaneous laryngeal reinnervation is one key to achieving this goal.

\section{BIBLIOGRAPHY}

1. Fried M, Meller SM, Rinaldo A. Adult laryngeal anatomy. In: Fried MP Ferlito A, eds. The Larynx, 3rd edition. San Diego, CA: Plural Publishing; 2009.

2. Crumley RL. Laryngeal synkinesis revisited. Ann Otol Rhinol Laryngol 2000;109:365-371.

3. Shindo ML, Herzon GD, Hanson DG, Cain DJ, Sahgal V. Effect of denervation on laryngeal muscles: a canine model. Laryngoscope 1992;102:663-669.

4. Blitzer A, Jahn AF, Keidar A. Semon's law revisited: an electromyographic analysis of laryngeal synkinesis. Ann Otol Rhinol Laryngol 1996;105:764-769.

5. Hirato I, Hirano M, Tomita H. Electromyographic investigation of human vocal cord paralysis. Ann Otol Rhinol Laryngol 1968;77:296-304.

6. Hogikyan ND, Johns MM, Kileny PR, Urbanchek M, Carroll WR, Kuzon WM. Motion-specific laryngeal reinnervation using muscle-nerve-muscle neurotization. Ann Otol Rhinol Laryngol 2001;110:801-810.

7. Johns MJ, Urbanchek M, Chepeha DB, Kuzon WM, Hogikyan ND. Thyroarytenoid muscle maintains normal contractile force in chronic vocal fold immobility. Laryngoscope 2001;111:2152-2156.
8. Flint PW, Downs DH, Coltrera MD. Laryngeal synkinesis following reinnervation in the rat. Neuroanatomic and physiologic study using retrograde fluorescent tracers and electromyography. Ann Otol Rhin Laryngol 1991;100:797-806.

9. Crumley RL, McCabe BF. Regeneration of the RLN. Otolaryngol Head Neck Surg 1982;90:442-447.

10. Nomoto M, Yoshihara T, Kanda T, Kaneko T. Synapse formation by autonomic nerves in the previously denervated neuromuscular junctions of the feline intrinsic laryngeal muscles. Brain Res 1991;539:276-286.

11. Nomoto M, Yoshihara T, Kanda T, Konno A, Kaneko T. Misdirected reinnervation in the feline intrinsic laryngeal muscles after long-term denervation. Acta Otolaryngol Suppl (Stockh) 1993;506:71-74.

12. Hydman J, Mattsson P. Collateral reinnervation by the superior laryngeal nerve after recurrent laryngeal nerve injury. Muscle Nerve 2008;38:1280-1289.

13. Old MO, Oh SS, Feldman EL, Hogikyan ND. Novel model to assess laryngeal function, innervation, and reinnervation. Ann Otol Rhinol Laryngol 2011;120:331-338.

14. Tessema B, Pitman MJ, Roark RM, Berzofsky C, Sharma S, Schaefer SD. Evaluation of functional recovery of recurrent laryngeal nerve using transoral laryngeal bipolar electromyography: a rat model. Ann Otol Rhinol Laryngol 2008;117:604-608.

15. Wu BL, Sanders I, Mu L, Biller HF. The human communicating nerve. An extension of the external superior laryngeal nerve that innervates the vocal cord. Arch Otolaryngol Head Neck Surg 1994;120:1321-1328.

16. Nasri S, Beizai P, Ye YM, Sercarz JA, Kim YM, Berke GS. Cross-innervation of the thyroarytenoid muscle by a branch from the external division of the superior laryngeal nerve. Ann Otol Rhinol Laryngol 1997; 106:594-598

17. Sanudo JR, Maranillo E, Leon X, Mirapeix RM, Orus C, Quer M. An anatomical study of anastomoses between laryngeal nerves. Laryngoscope 1999;109:983-987.

18. Maranillo E, Leon X, Quer M, Orus C, Sanudo JR. Is the external laryngeal nerve an exclusively motor nerve? The cricothyroid connection branch. Laryngoscope 2003;113:525-529.

19. Van Daele DJ, Cassell MD. Multiple forebrain systems converge on motor neurons innervating the thyroarytenoid muscle. Neuroscience 2009;162:501-524.

20. Takaoka T, Shiotani A, Saito K, et al. Neuronal re-juvenilization in the nucleus ambiguus after vagal nerve injury. Neurosci Res 2009;65:353-359.

21. McRae BR, Kincaid JC, Illing EA, et al. Local neurotoxins for prevention of laryngeal synkinesis after recurrent laryngeal nerve injury. Ann Otol Rhinol Laryngol 2009;118:887-893. 